\title{
EDITORIAL
}

\section{NÚMERO ESPECIAL ANTROPOLOGÍA BIOLÓGICA Y PALEOPATOLOGÍA}

\author{
Claudia M. Rojas-Sepúlveda, MSc, PhD \\ Departamento de Antropología \\ Facultad de Ciencias Humanas \\ Universidad Nacional de Colombia-Sede Bogotá \\ Astrid L. Perafán Ledezma, MSc, PhD \\ Programa de Antropología \\ Facultad de Humanidades \\ Universidad del Magdalena-Santa Marta
}

La Antropología Biológica colombiana pasa por un momento interesante en que los esfuerzos de algunos investigadores, tras años de trabajo, poco a poco comienzan a retomar la relevancia que, hacia la década de 1970, les fue negada (Rodríguez, 1996, 2008; Rojas-Sepúlveda, 2014; Tabares, Rosique, \& Delgado-Burbano, 2012). Uno de los indicadores del interés en el conocimiento que sobre el ser humano facilita la antropología biológica, es la organización de diversos eventos de corte internacional que se han realizado en el país.

Durante parte del año 2012 y todo el año 2013 estuvimos organizando la $\mathrm{V}$ Reunión de la Asociación de Paleopatología en Suramérica, Paleopathology Association Meeting in South America-PAMinSA V. La cual se llevó a cabo del 14 al 16 de agosto de 2013 en la Universidad del
Magdalena en Santa Marta, Colombia, congregando académicos e investigadores de diferentes partes del mundo para compartir sus avances de investigación, nuevas propuestas metodológicas y técnicas para abordar el estudio de las enfermedades en el pasado (Rojas-Sepúlveda \& Perafán, 2013).

Las ponencias centrales del PAMinSA V estuvieron a cargo de tres de las figuras más reconocidas en el ámbito: el Dr. Douglas Ubelaker (EEUU), la Dra. Jane Buikstra (EEUU) y la Dra. Sheila Mendonça de Souza (Brasil). El evento además permitió que 125 personas, entre los cuales se encontraban conferencistas de gran trayectoria académica e investigativa, así como nuevos investigadores y estudiantes universitarios en proceso de formación provenientes de diferentes instituciones y lugares de América Latina y el mun- 
do (Argentina, Brasil, Perú, Ecuador, Bolivia, Colombia, México, Estados Unidos, Canadá, España, Austria y Reino Unido), compartieran sus experiencias en el campo. De esta manera, se presentaron 33 afiches y 41 ponencias orales de equipos nacionales e internacionales.

Nueve simposios fueron coordinados por colegas que evaluaron cada una de las ponencias antes de ser presentadas en ellos, garantizando de esta manera la calidad de las mismas. Estos simposios se titularon: 1. Propuestas metodológicas y nuevas aplicaciones de técnicas radiológicas, histológicas y biomoleculares en Paleopatología (Mg. María Antonieta Corcione y Esp. Freddy Rodríguez, Colombia); 2. Biología, Naturaleza y Cultura (Dr. Carlos Serrano, México); 3. E1 impacto de las enfermedades infecciosas en las condiciones de salud de las sociedades antiguas de América (Dra. Lourdes Márquez y Dr. José Vicente Rodríguez, México y Colombia); 4. Salud y Transiciones (Mg. Bibiana Cadena y Mg. Juliana Gómez, Colombia); 5. La aplicación de la paleopatología en la labor pericial antropológica forense: retos y reflexiones vigentes $(\mathrm{Mg}$. Ana Carolina Guatame y Ant. Carolina Puerto, Colombia); 6. Paleopatología y zooarqueología (paleopatología animal) (Dr. Alfredo Altamirano Enciso, Perú); 7. La enfermedad de chagas antes del contacto (Dr. Adauto José Gonçalves de Araújo, Brasil); 8. Investigaciones recientes en cavidad bucal (Dr. Jorge Suby, Argentina) y 9. Investigaciones recientes en temáticas diversas (Dr. Alena Mayo y Dr. Timisay Monsalve, Brasil y Colombia).

La importante participación de estudiantes de pregrado en antropología de algunas universidades del país (particularmente de la Universidad de Caldas y de la Universidad del Magdalena) en calidad de asistentes, demostró que este campo de investigación de la antropología cada día en Colombia gana más interesados en formarse e investigar sobre temas relacionados con la bioarqueología, la paleopatología y la antropología forense; resaltando adicionalmente, la necesidad de fortalecer e incentivar la formación e investigación bioantropológica, bioarqueológica y forense en el país.

Lo que fue todo un desafío se convirtió en una gran satisfacción, pues la respuesta de los colegas fue muy positiva. Hoy tenemos el agrado de presentar otro de los frutos de esta importante experiencia en que se constituyó la organización del PAMinSA V: este número especial de la Revista Jangwa Pana el cual recoge siete de los trabajos presentados en el evento. Aprovechamos para agradecer aquí de manera especial a los autores de los mismos, por haber respondido a nuestra convocatoria, permitir por este medio la difusión de sus investigaciones y soportar pacientemente el largo proceso editorial. Complementan este número tres trabajos presentados en el marco del evento organizado por el colega Dr. Eduardo Forero, en la Universidad del Magdalena: el IX Seminario Internacional de Patrimonio; y dos trabajos de algunos miembros del Programa de Antropología de la Universidad del Magdalena.

El artículo de Douglas Ubelaker "La contribución de las alteraciones patológicas a la interpretación en Antropología forense" se constituye en un trabajo que proporciona a través del estudio de evidencias patológicas herramientas metodológicas y técnicas para la interpretación de información en contextos médico-legales para la identificación de restos óseos humanos. En este artículo, Ubelaker señala que las patologías se constituyen en un referente multifacético clave para la interpretación de los datos en contextos forenses, para lo cual parte de la definición misma de enfermedad. Ésta tiene dos acepciones diferentes, la primera hace referencia a la enfermedad y sus efectos, frente a lo cual puede presentarse ambigüedades, ya que en algunos casos una misma enfermedad puede producir diferentes efectos en los restos óseos o varias enfermedades pueden producir efectos similares. La segunda definición de patología hace referencia a "algo anormal". En este caso, la identificación positiva en contextos médico-legales está articu- 
lada con el hallazgo de rasgos únicos e inusuales en los restos óseos asociados a alteraciones anormales que pudieron sufrir los individuos antes de su muerte o en un momento cercano a ella. Para contribuir en el esclarecimiento y aportes de la paleopatología, Ubelaker señala la importancia de diferenciar entre "trauma perimortem", como aquellas evidencias que se producen alrededor de la muerte, aquellas "antemortem" las cuales se producen cierto tiempo antes de la muerte y las modificaciones "postmortem" producidas con posterioridad al deceso. De esta manera, el trauma perimortem puede asociarse con las causas de muerte y en este punto, el artículo describe los diversos mecanismos los pueden producir junto con magníficos ejemplos que permiten identificar patrones asociados a estos. La importancia de la detección de alteraciones que se pueden producir en los huesos de forma postmortem, en este caso por efectos tafonómicos o por traumas que acontecen después de la muerte o pseudotraumas, es claramente subrayada por el autor. Igualmente, hace visible la importancia de la identificación a través de la variación anatómica normal y anormal y en esta medida la identificación positiva a través de rasgos anatómicos compartidos y de la singularidad del rasgo compartido, ejemplo de ello es el éxito en la identificación que se puede realizar a través de la presencia de rastros de enfermedades o de tratamientos para curar las mismas apoyándose en el uso de archivos médicos familiares o en análisis moleculares especializados. En fin, el artículo recoge parte de la experiencia del autor quien a través de la presentación de casos concretos justifica la gran utilidad de la paleopatología en el ámbito forense.

El artículo de Fabra, Salega, González y Tavorone, titulado "Lo que el agua nos dejó: una investigación bioarqueológica en la costa de la Laguna Mar Chiquita (Córdoba - Argentina)", da cuenta de 8 sitios arqueológicos costeros de la Laguna Mar Chiquita, noreste de Córdoba Argentina (4058 +-89 t 487 +- 45 años AP) resultado de excavaciones de rescate adelantadas por el Programa de Arqueología Pública-PAP- (SEI,
Museo de Antropología, FfyH, UNC) en los cuales se identificaron restos óseos de 11 individuos adultos. Se presenta en el artículo las patologías por ellos sufridas y la reconstrucción de las prácticas mortuorias, atendiendo de manera cuidadosa a la metodología utilizada en los procesos de excavación y en el análisis paleopatológico. Para indagar sobre las enfermedades los autores tuvieron en cuenta indicadores dentales (caries, abscesos y pérdidas dentales antemortem, hipoplasia del esmalte) y óseos asociadas con actividades físicas (nódulos de Schmorl en la columna vertebral, eburnación en las superficies articulares del esqueleto apendicular y ostefitosis). A partir de los resultados obtenidos, los investigadores evidencian en la muestra ósea una prevalencia baja y moderada de caries dental, hipoplasia del esmalte y pérdidas antemortem, lo cual está asociado con poblaciones con una economía cazadora-recolectora o mixta. Un aspecto a destacar es la presencia de un enterramiento novedoso para la región (posición sedente) no reportado previamente y que permite a los investigadores plantear la existencia de dinámicas sociales complejas para esta población que habitó el centro de Argentina en el Holoceno Medio. De igual manera, el artículo reporta otro aspecto destacable como lo es la presencia de un proyectil de cuarzo asociado ${ }^{\text {a }}$ uno de los individuos analizados y que se lo relaciona con violencia interpersonal que podría ser consecuencia de una disminución de la calidad de vida en el área hacia el Holoceno final tardío (1000-500 años AP), causada por un incremento demográfico y una mayor competencia para accedes a los recursos disponibles.

El artículo "La Danza de los esqueletos: la enseñanza de la bioarqueología a los niños y al público en general" de Sandra Assis y Ana Luisa Santos, presenta la valiosa experiencia realizada por el Grupo de Estudio Evolución Humana del Departamento de Ciencias de la Vida de la Universidad de Coimbra en escuelas públicas de Primaria y Secundaria, así como en museos, organismos municipales y otros grupos escolares, en Portugal y en el extranjero, con el fin de 
transferir de manera lúdica conocimientos sobre antropología biológica, biocultural y evolución humana. La transferencia de conocimiento se realizó a través del taller "La danza de los esqueletos" en el marco del Proyecto "Jugar con el Gran Árbol de la Evolución”, el cual contó con apoyo gubernamental. En el artículo se hace énfasis en que el taller "La danza de los esqueletos" permitió difundir la importancia del estudio del esqueleto humano en contextos arqueológicos, introduciendo a los estudiantes en el trabajo de campo y en el laboratorio bioantropológico donde se enfatizó en la gran cantidad de información que los restos óseos pueden proporcionar (sexo, edad, patologías e historias de vida). En el artículo además se describe parte del taller, la cual consiste en el juego "La danza de los esqueletos", el cual se desarrolla en equipos y permite la participación interactiva de estudiantes, docentes y moderadores. Estos talleres permitieron además introducir a los estudiantes en el conocimiento de la bioarqueología, un área poco conocida en la región. Los investigadores pudieron encontrar en la experiencia una importante aceptación por parte del público y la posibilidad de plantear este tipo de enseñanza no formal como complementara con planes de estudio establecidos.

En el artículo "Salud en el Horizonte Tardío: Pastores y agricultores de Pueblo Viejo Pucará y Huamanmarca (Perú)", Kolp-Godoy, Palma, Enríquez, Fernández y Makowski analizan el estado de salud y nutrición de dos asentamientos andinos del Horizonte Tardío (1430-1532 DC) procedentes de la Costa y Sierra de Lima, Pueblo Viejo Pucará (PVP) (pueblo pastoril) y Huamanmarca (HVA) (comunidad agraria) a través del estudio de nueve marcadores bioarqueológicos dentales y óseos: caries dental, desgaste oclusodental, sarro dental, hipercementosis, criba orbitalia, hiperostosis porótica, periostitis y trauma óseo. De manera interesante, los resultados obtenidos permiten a los autores concluir que existe una asociación entre los indicadores de estrés no específico, los marcadores dentales, el nivel de salud y nutrición de la población, con la procedencia, el estatus social y la actividad ocupacional de los habitantes. Los niños y los adultos del PVP del sector I dedicados al pastoreo y domesticación de camélidos y las mujeres dedicadas a actividades artesanales y agrícolas, fueron los más propensos a padecer enfermedades bucodentales y a tener una salud pobre; por su parte, los niños presentaron desnutrición y procesos bacterianos infecciosos. En el sitio HVA fueron los hombres y las mujeres adultos del sector II los que padecieron estrés mecánico (fracturas óseas) asociados a largas jornadas de trabajo.

Liliana Serrano y Ana Luisa Santos en su artículo "Minería en Aljustrel (Portugal): salud, enfermedad y actividades en los habitantes del pasado", dan cuenta del estudio de las patologías presentes en restos óseos humanos de adultos encontrados en la necrópolis de S. Bartolomeu Aljustrel, pertenecientes al período comprendido entre el siglo VII y el siglo XVII AD. Dichos restos fueron excavados entre 1981 y 1982 y analizados en el año 2013 para inferir la relación entre las actividades ocupacionales relacionadas con la minería y las patologías óseas. La muestra fue analizada macroscópicamente para identificar el sexo, estimar la edad e identificar las patologías ( orales, traumáticas, infecciosas y congénitas). El estudio evidenció una baja prevalencia de lesiones cariogénicas en individuos a quienes no fue posible identificar el sexo y lesiones traumáticas en costillas, ulnas y fíbulas en individuos masculinos. A pesar de que las lesiones identificadas no pudieron ser asociadas directamente con la actividad minera, las autoras registraron alteraciones morfológicas como la eversión del ángulo goníaco que podría relacionarse con el transporte de cargas pesadas en la cabeza y la hiperdorsiflexión de la articulación metatarsofalángica, que podría asociarse con el acuclillamiento.

En el artículo "Mujeres del formativo. Salud y actividades en San José Mogote, Oaxaca, México" Martha Alfaro y Susana Gómez buscan aproximarse a algunos aspectos de la cotidianidad de las mujeres que habitaron la aldea Formativa de San 
José Mogote (1400 a.C. a 200 a.C.) e identificar factores que pudieron afectar su salud a través del análisis de patologías e indicadores de estrés ocupacional, datos etnohistóricos, arqueológicos y etnográficos de la región. Las alteraciones patológicas que evaluaron fueron: criba orbitaria, hiperostosis porótica, hipoplasia del esmalte, patologías bucales, procesos infecciosos, lesiones degenerativas de tejido articular y traumatismos, además desgate dental no relacionado con la alimentación. Las autoras evidenciaron una baja incidencia de criba, hiperostosis porótica e hipoplasia del esmalte, hallando que su distribución se asocia con el sexo, al presentarse una leve superioridad de presencia de estas enfermedades en las mujeres en relación con los hombres. El estudio aporta evidencias sobre las actividades de subsistencia en dicho período, indicando que combinaba la caza, la recolección y la agricultura. Lo observado en este trabajo muestra que en la región se dio por aquella época un período de crecimiento político y económico. La mayor frecuencia de caries en mujeres se asoció con las actividades que ellas realizaban, la recolección de plantas y de semillas, lo cual probablemente les permitió unmayor consumo de carbohidratos. Las lesiones degenerativas en mujeres se presentaron en el codo, zona torácica y lumbar y se asociaron con actividades como la molienda en metate, elaboración de cerámica, cestería y tejido. En los hombres, se observó en mayor medida la presencia de lesiones degenerativas en hombro, codo, mano y en las regiones lumbar y cervical de la columna, lo cual se relacionó con actividades de caza y recolección y la carga de grandes pesos en la espalda. Se encontraron pocas lesiones traumáticas asociadas con caídas y poco desgaste dental resultado del uso de los dientes como herramienta.

El artículo "El Trauma en la piel: un análisis paleopatológico de tatuajes Paracas- Necrópolis" presentado por Patricia Maita y Enma Minaya Cabello, analiza las consecuencias paleopatológicas del tatuaje en un grupo de momias del periodo Paracas Necrópolis procedentes del ce- menterio de Warikayan (500 a.C -100 d.C) en la costa sur del Perú. Para el análisis bioarqueológico, las autoras utilizaron técnicas novedosas en bioarqueología, por un lado, microscopia óptica y fluorescencia de rayos X con el fin de caracterizar los pigmentos insertos en la piel, y por el otro, refectografía infrarroja, para localizar las lesiones tegumentarias ocasionadas por la modificación cultural del cuerpo. En total analizaron 20 momias que presentan tejido momificado con tatuajes. Los individuos femeninos incluidos en el estudio presentan más tatuajes y mayores áreas corporales tatuadas en relación con los hombres. Las autoras identificaron diseños diferentes de tatuajes (zoomorfos, símbolos geométricos como puntos, líneas y círculos, figuras abstractas, figuras como ser oculado, orca asesina y la greca escalonada) los cuales son idénticos a la iconografía registrada en la cerámica y en textiles de estilo Paracas Necrópolis. El material utilizado para la realización de los tatuajes fue el hollín. Estos se realizaron principalmente en las manos, dedos, antebrazo, miembros inferiores y la cara, siendo los miembros inferiores las regiones con mayor número de tatuajes, y la cara, el área anatómica menos tatuada. El análisis aquí presentado indica que los tatuajes fueron realizados con mucha antelación a la muerte del individuo. Debido a que no se hallaron cicatrices en la piel, el estudio no pudo determinar la técnica utilizada para el tatuado. De igual forma, no evidenció alteraciones dérmicas asociadas a los tatuajes que sugirieran algún tipo de reacción o enfermedad.

Posteriormente, este número especial de la Revista Jangwa Pana cuenta con un grupo de tres artículos sobre patrimonio. El primero se titula "El patrimonio como espacio de conflicto en Barichara- Santander" presentado por Luz Andrea Cote, artículo en el cual la autora reflexiona sobre la conflictividad propia del campo del patrimonio cultural, así como los impactos socioculturales de los procesos de patrimonialización. La autora concluye que en Barichara, el patrimonio cultural entra en conflicto, ya que se trata de una "cultura viva" ya que está en constan- 
te práctica y genera una renovación permanente del conocimiento. Además, el artículo enfatiza en que la identidad cultural y el patrimonio cultural son resultados de la mirada de múltiples actores públicos y privados, dentro de los cuales se encuentran los "nuevos vecinos". La presencia de estos últimos ha generado tensiones entre estos y los lugareños, las cuales reposan en los cambios en relación con el patrimonio, con los oficios, con la pérdida de control y con la toma de decisiones. El segundo artículo "La noción del paisaje en arqueología. Formas de Estudio y aportes al patrimonio" de Inés Gordillo busca abordar el paisaje desde la arqueología y otras disciplinas como la geografía humanística en el ámbito de la gestión ambiental. La autora reflexiona sobre la manera cómo el paisaje es entendido, en términos relacionales y holísticos, como una realidad social históricamente construida y que está determinada por relaciones de poder estructuradas en relación con la edad, el género y la posición social. El espacio es entonces considerado como un espacio polisémico, el cual se vive, se piensa, se imagina, y es objeto y representación. El último artículo de este bloque "La nación Unificada en el escenario. Sonia Osorio y el Carnaval de Barranquilla" de María Teresa García, da cuenta de cómo Sonia Osorio, a través de la mezcla de la estética del ballet (propuesta estética de las élites de Barranquilla) con la del folklor del carnaval de Barranquilla (exhibicionismo sexual), logra crear un discurso unificado de la nación heterosexual fragmentada. Al integrar en su espectáculo danzas de diferentes partes del país y al mismo tiempo transgredirlo al darle "casa" a la comparsa de la "calle", Sonia Osorio permitió el paso de un dominio a otro como nación/mundo, pobre/ rico, virgen/reina, a la vez, entrelazó danza, nación y reinas con poderes regionales y articuló las apuestas nacionales con el mercado global. El artículo finalmente reflexiona sobre el papel de Sonia Osorio en el tránsito de algunos sujetos con capitales culturales desvalorizados como las mujeres negras, hacia ámbitos diferentes a través de la danza, pasando de lo doméstico a lo univer- sal y de ser esencializadas y naturalizadas en lo erótico, a ser reinas - divas.

Por último, este número especial de la Revista Jangwa Pana incluye dos artículos presentados por un docente y dos estudiantes del Programa de Antropología de la Universidad del Magdalena. El primero, "Innovación Popular para acceder al agua: tecnología, creatividad y organización comunitaria en el Barrio Luis R. Calvo (Santa Marta)" de Raiza Llinás y William Andrés Martínez, explora el concepto de innovación popular y lo hace visible a través de la forma cómo las innovaciones populares son creadas por la población desplazada que habita en el Barrio Luis R. Calvo en Santa Marta (Colombia) para resolver sus dificultades para acceder al agua potable. En el artículo los autores presentan cómo dichas innovaciones se constituyen en entramados socio-técnicos donde la comunidad de este barrio y la tecnología se coproducen generando conexiones con el territorio, lo que permite también crear y fortalecer redes de solidaridad social. El último artículo, de autoría de Jhonatan Porto, "Afrodescendientes en Colombia: una revisión de los últimos seis años" aborda los diferentes estudios que se han adelantado en relación con los afrocolombianos desde 2008, fecha de publicación de una exhaustiva revisión, hasta el 2013. El artículo da cuenta de cómo la población afrodescendiente en nuestro país ha estado expuesta por largos períodos de tiempo a situaciones de violencia, pobreza, marginalidad, inestabilidad económica y rechazo.

En fin, este número especial de la Revista Jangwa Pana cuenta con una cuidadosa selección de trabajos que con seguridad serán de interés de la comunidad académica antropológica y en particular, de la comunidad académica dedicada a la antropología biocultural y a la paleopatología.

Teniendo en cuenta que los trabajos aquí presentados surgen del PAMinSA V, es para nosotras vital agradecer a aquellas personas e ins- 
tituciones que hicieron posible dicho evento. Agradecemos entonces de manera muy especial a los tres conferencistas centrales del evento quienes muy generosamente aportaron al éxito del mismo: Dr. Douglas Ubelaker (EEUU), Dr. Jane Buikstra (EEUU) y Dr. Sheila Mendonca de Souza (Brasil). Igualmente, a los miembros del Comité Científico, quienes aseguraron la rigurosidad del evento: Dr. Adauto Araújo (Brasil); Dr. Bernardo Arriaza (Chile); Dra. Jane Buikstra (EEUU); Dra. Della Cook (EEUU); Dr. Ricardo Guichón (Argentina); Dra. Sonia Guillén (Perú); Dr. Clark Spencer Larsen (EEUU); Dra. Lourdes Márquez (México); Dra. Sheila Mendonca de Souza (Brasil); Dra. Elizabeth Ramos (Colombia); Dra. Charlotte Roberts (Reino Unido); Dr. José Vicente Rodríguez (Colombia); Dr. Carlos Serrano (México); Dr. Jorge Suby (Argentina); Dr. Douglas Ubelaker (EEUU); Dr. John Verano (EEUU). Mil gracias a los profesores y sus instituciones: Dr. José Vicente Rodríguez (Universidad Nacional de Colombia), Dra. Elizabeth Ramos (Universidad del Cauca), Ant. César González (Universidad de Caldas) y Dr. Juan Guillermo Martín (Universidad del Norte), por sus aportes como coorganizadores. A la Universidad del Magdalena por sus aportes en capacidad instalada como institución organizadora. Igualmente, agradecemos especialemente a la Fundación de Investigaciones Arqueológicas Nacionales por su aporte para financiar el evento, a la Paleopathology Association y su presidenta, la Dra. Charlotte Roberts, por el constante apoyo en la organización y a la Federación Nacional de Cafeteros de Colombia, por su aporte al evento. Un agradecimiento muy especial a los colegas que evaluaron con tanta rigurosidad los trabajos que aquí se presentan.

\section{Referencias Bibliográficas}

Rodríguez, J. V. (1996). Panorama de la Antropología Biológica en Colombia y su relación con el ámbito latinoamericano y mundial. Maguaré, 11-12, 75-102.

Rodríguez, J. V. (2008). El desarrollo de la antropología biológica en Colombia. Colantropos, http://www(Consultado el 1 de septiembre de 2013).

Rojas-Sepúlveda, C. (2014). Breve historia, balance y perspectivas de la bioarqueología en Colombia. In L. Luna, C. Aranda, \& J. Suby (Eds.), Avances recientes en la bioarqueología latinoamericana (p. En prensa).

Rojas-Sepúlveda, C., \& Perafán, A(Eds). (2013). Libro de resúmenes de la V Paleopathology Association Meeting in South AmericaPaminsa V. Santa Marta, Colombia.

Tabares, E., Rosique, J., \& Delgado-Burbano, M. (2012). Tendencia de la bioantropología y un estudio de caso: su desarrollo académico en la Universidad del Cauca. Revista Colombiana de Antropología 2, 48(1), 259-278. 